\title{
La gestión del desarrollo regional en Cuba. Un enfoque desde la endogeneidad
}

\author{
RAMÓN GONZÁLEZ FONTES* \\ ANa DE Dios MarTíneZ** \\ Rafael Montejo VÉLIZ***
}

\begin{abstract}
This paper examines the central elements of the endogenous development theory under the point of view of the current singular conditions of Cuban economy. From this unique confrontation comes to be what we call endogenous regional development management. Endogenicness means a combination of the conditions of development provided by national sources and the full exploitation of the potentials of territory development. So, in the case of Cuban economy, a new profile in the management of the territorial development emerges.
\end{abstract}

Keywords: development, endogenous development, synergies, Cuba, local development.

\section{Resumen}

Este trabajo examina los elementos centrales de la teoría del desarrollo endógeno a la luz de las condiciones actuales de la economía cubana. De esta singular confrontación emana lo que los autores denominamos gestión del desarrollo regional desde la endogeneidad. La endogeneidad significa una combinación de las condicionantes de desarrollo que surgen de las fuentes nacionales y la más plena utilización del potencial de desarrollo del territorio. Se perfila así un nuevo enfoque de gestión de desarrollo del territorio para el caso de la economía cubana.

Palabras clave: desarrollo, desarrollo endógeno, sinergias, Cuba, desarrollo local.

\footnotetext{
* Universidad de Camagüey, Cuba. Correo-e: rglez2002@yahoo.com

** Universidad de Camagüey, Cuba. Correo-e: ana@reduc.cmw.edu.cu

*** Universidad de Camagüey, Cuba. Correo-e: mveliz@reduc.cmw.edu.cu
} 


\section{Introducción}

Los problemas del desarrollo han constituido, en los últimos cincuenta años, el centro de atención de políticos, estadistas, académicos y científicos de diversas partes del planeta, debido a que ha ido cobrando fuerza la idea de que en su solución se define la propia existencia de la humanidad. Esto ha provocado que desde el punto de vista conceptual se haya acumulado un notable avance teórico del estudio de esta problemática y que, de forma práctica, se produzca un gran arsenal de modelos y estrategias con la pretensión de obtener respuestas.

En este sentido, la Cuba de la época revolucionaria no ha sido una excepción y los cuarenta años transcurridos a partir de 1959 han sido testigos de un notable esfuerzo en la búsqueda de soluciones y en el establecimiento y defensa del proyecto político que se definió desde 1953 en La historia me absolverá, ${ }^{1}$ y que fue rediseñándose a partir de 1961 con la declaración del carácter socialista de la revolución (Castro, 1971, 2). En todo este periodo se ha trabajado para lograr lo que se definió como el desarrollo integral del país (Rodríguez, 1983, 18), y que ha estado orientado a solventar las graves deformaciones estructurales, heredadas tras varios siglos de dominación colonial y neocolonial.

El final de la década de los ochenta y toda la década de los noventa ha sido un periodo caracterizado por un notable esfuerzo para sobreponerse a una de las crisis económicas mas profundas de su historia, ${ }^{2}$ y por una búsqueda constante de soluciones a los problemas que se han ido presentando. Esta crisis tiene sus causas en la transformación del escenario político, económico y social del mundo, a partir de la desintegración de la URSS y la desaparición del campo socialista; el recrudecimiento del bloqueo y la guerra económica de los Estados Unidos de Norteamérica, todo lo cual agotó las posibilidades del sistema centralizado de dirección y planificación de la economía que se venía aplicando desde 1975, y que se sustentaba en las relaciones de Cuba con el Consejo de Ayuda Mutua Económica (CAME).

Es por ello que en la actualidad constituye un objetivo primordial la búsqueda de soluciones a los problemas que enfrenta

${ }^{1}$ Alegato de autodefensa presentado por Fidel Castro en el juicio por el asalto al cuartel Moncada de Santiago de Cuba, el 26 de julio de 1953, y que constituye el primer programa político de la época revolucionaria, conocido también como El Programa del Moncada.

${ }^{2}$ Hasta 1993 el Producto Interno Bruto cubano disminuyó 35\% con relación a 1989. 
el país, apoyándose en sus propias posibilidades, considerando el aislamiento económico a que está siendo sometido y por la necesidad de que dichas soluciones tengan un carácter irreversible y autosostenible.

El objetivo de este trabajo es mostrar, para el caso de Cuba, una concepción del desarrollo territorial que hace énfasis en su potencial endógeno, sobre la base de la participación activa de los gobiernos locales en la solución de los problemas y brechas del desarrollo del país. La síntesis conceptual de este trabajo toma en consideración los fundamentos y mecanismos desarrollados en la teoría contemporánea del desarrollo endógeno, adecuadas a las realidades y exigencias del sistema institucional y socioeconómico cubano, sobre la base de los resultados de un conjunto de investigaciones realizadas por los autores. Este enfoque aporta a la concepción tradicional de la teoría y la práctica del desarrollo endógeno, en primer lugar, el reconocimiento explícito de la necesidad de una gestión del desarrollo, y en segundo lugar, que dicha gestión -sobre todo en atención al carácter subdesarrollado del país- se realice desde los territorios, sobre la base de estrategias territoriales en combinación armónica y complementada con las fuentes y factores de desarrollo que emanen de las estrategias y políticas nacionales.

Es evidente, por supuesto, que la problemática analizada y las soluciones planteadas se han elaborado para las condiciones concretas del caso cubano, sin pretender realizar extrapolaciones a otras realidades, en las que no podrían resultar viables; sin embargo, estos resultados indican direcciones de futuras investigaciones, que podrían dar paso a soluciones más generales.

\section{El desarrollo y su gestión desde una perspectiva territorial}

La gestión del desarrollo de los sistemas empresariales ha sido ampliamente estudiada, sobre la base de su funcionamiento bajo las condiciones de los diferentes sistemas socioeconómicos, de la economía de mercado o de dirección centralizada, acerca de lo cual existe una amplia literatura.

A diferencia del campo empresarial, no existe claridad cuando se trata de la gestión del desarrollo regional; esto se debe a que su definición y estructura conceptual está cargada de enfoques normativos que condicionan, a priori, las vías y procedimientos de gestión, y no permiten poner en claro cuáles son los aspectos clave sobre los que deberá recaer de forma prioritaria la 
acción de gestión. Esta problemática se debe, fundamentalmente, a que el propio concepto de desarrollo ha llevado implícito siempre una versión sobre lo que es deseable o no para un sistema nacional o territorial, por estar basado en principios valorativos, éticos y normativos de lo que conviene o no para el ser humano (Todaro, 1984, 21). No obstante, en los últimos años se han ido despejando cuáles son los aspectos esenciales de estos procesos, independientemente del marco institucional ${ }^{3}$ en el cual se manifiesten.

Si se analizan algunas de las concepciones contemporáneas del desarrollo, se puede apreciar que han venido experimentando cambios sustanciales después de la Segunda Guerra Mundial, con una marcada tendencia a poner al ser humano en el centro de atención del problema, en íntima relación con el medio, ya que, en última instancia, es el hombre quien padece o se beneficia de la situación social.

Desde finales de la década de los ochenta y toda la década de los noventa continuó desarrollándose esta concepción humanista del desarrollo, aunque condicionada en sus aspectos valorativos de políticas y de estrategias para alcanzarlo, en correspondencia con la envoltura institucional de las sociedades donde se aplican, mientras que los juicios de valor de los que realizan los análisis toman las decisiones y elaboran las políticas y estrategias de desarrollo (CIEM, 1996: 20-24).

De acuerdo con los objetivos que se pretenden lograr, lo importante de todo este desarrollo conceptual es que pueden aclararse cuáles son los elementos esenciales para que exista desarrollo, o sea, se debe centrar la atención en el bienestar del ser humano en equilibrio con el entorno natural y en la posibilidad de aumentar constantemente las oportunidades de reforzar esa condición humana, aunque su logro y alcance dependan de la posición relativa en que se encuentren los territorios analizados en relación con sistemas territoriales de referencia, y de los análisis

${ }^{3}$ En su obra Institutions, institutional change and economic performance, Douglas North define y fundamenta cómo las instituciones constituyen las reglas de juego del funcionamiento de una sociedad y demuestra cómo las mismas influyen en el desempeño de la economía (North, 1998). A esto se puede añadir que las instituciones, las relaciones institucionales o el marco institucional se encuentran determinadas en última instancia por las relaciones sociales de producción y su esencia, las relaciones de propiedad, que caracterizan a la organización económica o modo de producción, pudiendo encontrarlas reflejadas tanto en las relaciones de producción (relaciones de propiedad, o relaciones de mercado, etcétera), como en los elementos de la superestructura que a ellas le corresponden. 
valorativos que se hagan o se tengan de lo que es bueno o no, y de cómo debe estar distribuido este bienestar.

\subsection{Las fuentes del desarrollo en los territorios}

En este nivel de análisis resulta imprescindible aislar los elementos que pueden constituir las fuentes de cualquier proceso de desarrollo. Así, el desarrollo es una combinación del bienestar que proporciona el disfrute de determinados niveles de consumo actual y el progreso que garantiza los niveles de acumulación para proporcionar el bienestar futuro, en armonía con el entorno.

El punto de partida de este proceso para un espacio dado, lo constituye el conjunto de recursos (naturales, históricos, culturales, humanos, tecnológicos, económicos, institucionales y materiales) que forman su potencial de desarrollo (Vázquez Barquero, 1999, 23). ${ }^{4} \mathrm{Al}$ poseer un carácter histórico concreto, este punto de partida provoca que tanto cuantitativa como cualitativamente sea diferente para los distintos países o regiones. Sin embargo, desde el punto de vista conceptual, constituye la capacidad de generar una determinada cantidad de riqueza (material, humana, ${ }^{5}$ cultural o espiritual), que garantice la elevación del bienestar actual y del progreso que va a dar respuesta futura al crecimiento de la población, de las necesidades y a retroalimentar el propio proceso de cambio de forma continua y sostenible.

En función de cómo sea ese potencial y de la organización del sistema institucional bajo el cual se ejecute el proceso de obtención de esa riqueza, así serán los resultados del desarrollo del territorio. Es decir, que puede ser abundante y bien organizado, o llegar a estar tan deprimido y desorganizado que exista una ausencia de desarrollo o de sustentabilidad, o cualquier otra combinación de estas situaciones. Evidentemente, el problema de la gestión del desarrollo radica en establecer cómo y de dónde puede provenir el logro de magnitudes excedentes de riqueza que alimenten o complementen, en cualquier caso, este potencial.

Así, la primera fuente de desarrollo es el potencial que posee el sistema territorial. Esta fuente originaria puede ser alimentada por otras, desde dos direcciones diferentes, pero que se com-

${ }^{4}$ Sobre el concepto de potencial de desarrollo véase el trabajo de Vázquez Barquero (1999), donde plantea el criterio de que dicho potencial es el punto de partida para cualquier proceso de desarrollo.

${ }^{5}$ Cuando se habla de riqueza humana, se refiere no sólo a la posibilidad de incrementar cuantitativamente la población o los recursos laborales, sino también su preparación técnica y cultural como capital humano. 
plementan. Una tiene un carácter exógeno y viene dada por la corriente de riqueza (histórica, cultural, humana, tecnológica, económica, institucional y material), que viniendo desde fuera del sistema nacional o territorial, se integra a éste y es utilizado para ampliar en el territorio el bienestar, el progreso del ser humano, o ambos inclusive, gracias a los mecanismos de redistribución que existan en sistemas de orden superior, o a los que se logren por la atractividad del territorio. ${ }^{6}$ La otra fuente tiene un carácter endógeno, mediante el cual el propio sistema es capaz de generarlo o incrementarlo desde dentro, gracias a la coherencia de su organización, que le permite aprovechar toda la sinergia $^{7}$ que es capaz de desplegar y que se encuentra latente en su capacidad interna (Vázquez Barquero, 2000).

Queda evidenciado, pues, que los elementos anteriormente analizados no dependen de los juicios de valor con que sean interrelacionados o utilizados, aunque estos últimos y las formas organizativas que adopten (Saxenian 1998, 20) sí determinen y condicionen el resultado final y sus interrelaciones, ya que como resulta comprensible, estos elementos esenciales alcanzan determinadas magnitudes, toman determinados rumbos y producen efectos e impactos sobre unos y otros sistemas nacionales y territoriales, en dependencia de la envoltura institucional que los alberga e interrelaciona y las formas organizativas que internamente adopten.

No se pretende hacer una demostración exhaustiva de la eficiencia o deficiencias que los diferentes sistemas institucionales de la sociedad puedan jugar o hayan jugado en el actual estado de cosas con relación al desarrollo en los territorios. Nos limitaremos simplemente a apuntar que existen evidencias empíricas irrefutables de que la historia de la humanidad ha dibujado un sistema mundial extraordinariamente desigual y en franco proceso de deterioro, ${ }^{8}$ tanto a escala internacional como hacia los es-

${ }^{6} \mathrm{La}$ atractividad del territorio es su capacidad de concitar el interés por parte de las empresas nacionales y extranjeras para invertir en el territorio. Expuesto por De Mattos $(1999,7)$.

${ }^{7}$ El término sinergia se utiliza aquí como el incremento de la acción de dos elementos de un sistema cuando actúan conjuntamente o cuando por el actuar de elementos del sistema se produce una exaltación recíproca del efecto que producen dichos elementos y su relación en el sistema. Un elemento esencial de la materialización de la capacidad de desarrollo de un territorio endógeno radica básicamente en su capacidad de desarrollar fuentes de sinergias que se logran en los sistemas territoriales.

${ }^{8}$ Baste señalar que hasta septiembre de 1998 "El 86\% del consumo mundial actual corresponde a sólo el $20 \%$ de la población del mundo. Muchos millones de personas, en particular en el mundo en desarrollo, han sido excluidas por la reducción del consu- 
pacios subnacionales. Esto se ha debido a un condicionamiento histórico concreto de partida, y a que los sistemas de explotación imperantes, sobre todo el capitalista, han profundizado esta desigualdad.

Esta situación ha deprimido el potencial de desarrollo de muchos países y territorios, hasta hacerlo prácticamente inexistente, y con ello también la posibilidad de materializar y fomentar su propia capacidad endógena de desarrollo y la capacidad de atracción de fuentes exógenas, las que, además, se dirigen hacia los lugares más atractivos, en correspondencia con la dinámica del capital.

Tanto a escala mundial como hacia el interior de los países existen territorios muy deprimidos, lo que requiere que existan mecanismos de cooperación, intercambio, distribución y redistribución que permitan elevar el potencial de desarrollo de los mismos a partir de procesos exógenos, para con ello poder emplear y desplegar su capacidad endógena, ya que el desarrollo es producto del potencial de cada territorio y del efecto conjunto de estas dos fuentes, pues ninguna de ellas debe considerarse como absoluta.

Por ello, cuando se habla de utilizar la capacidad endógena de los territorios, no se puede pasar por alto el punto de partida que tiene cada cual, ya que resulta ilusorio pensar que el propio proceso de globalización de la economía va a generar de forma espontánea una respuesta productiva en todos los territorios, que genere de la nada un sistema productivo organizado y tecnológicamente avanzado capaz de insertarse competitivamente en los circuitos de acumulación del capital.

En este proceso, de manera espontánea habrá territorios perdedores que en el sentido endógeno no tendrán la posibilidad de estructurar sus fuentes de sinergia de tal manera que generen procesos de desarrollo, lo cual constituye algo muy relevante y la teoría contemporánea del desarrollo endógeno no le ha otorgado la debida atención.

Por ello un elemento vital de todos estos procesos lo constituyen los distintos flujos de riquezas que se producen y circulan a través de los distintos territorios, así como la capacidad que adquiere cada territorio o nación de reinvertir parte de esas ri-

mo. En algunos países la gente tiene menos bienes y servicios a su disposición que los que tenía hace 20 o 30 años" (Nota introductoria de Ariel François, representante residente del PNUD en Cuba en el Informe sobre Desarrollo Humano 1998.) (PNUD, 1990). 
quezas en incrementar el bienestar o el nivel de progreso de su población y territorio.

Todo esto quiere decir que, en ocasiones, la capacidad endógena no se manifiesta y requiere un impulso exógeno para entrar en acción; pero la influencia exógena requiere de una organización adecuada del sistema territorial hacia su interior, ya que sólo así podrá aprovechar al máximo la capacidad de incremento del potencial, que pueda ser capaz de provocar dicha influencia externa, evitando que las mismas se conviertan en una vía de extracción de las riquezas existentes en el potencial de desarrollo del territorio.

La gestión del desarrollo debe guiarse por la utilización regulada, armónica y combinada de todas las fuentes de desarrollo, ya que de lo contrario la fuente exógena podría crear una situación de deformación estructural en el territorio. Por ello debe tenerse presente que las regiones que asimilen inversiones o flujos de recursos externos deberán atender aspectos clave, tal como los resume Vázquez Barquero (1999):

- Que las inversiones o empresas puedan convertirse en polos de crecimiento y no se conviertan en enclaves económicos, con escasos o ningún vínculo con el sistema productivo territorial;

- Que estas inversiones no se conviertan sólo en polos de absorción de los recursos empresariales locales, privándolo de la capacidad técnica que le permitiría encausar su desarrollo y con ello el del territorio en su conjunto;

- Que estos flujos no ocupen una posición subordinada en cuanto a las inversiones en investigación y desarrollo y que ésta no se realice solamente en las casas matrices ubicadas fuera del territorio.

- A lo que se debe añadir aquí, que los flujos financieros que se producen en el territorio puedan ser aprovechados, en alguna medida, por los propios territorios en el desarrollo de los mismos.

Con ello no se pretende reducir el papel de las corrientes exógenas en el desarrollo de los territorios, sino que su proceso de gestión ha de requerir un territorio protegido y organizado, para que estas corrientes estimulen el cambio y no se conviertan en un factor de dependencia y deterioro.

Ante estos retos, resulta poco probable que territorios periféricos, aislados y desprotegidos puedan gestionar y crear, por sí solos, una capacidad de respuesta ante los retos de la globaliza- 
ción e insertarse competitivamente en este mundo global, lo que da lugar a lo que se reconoce como la gestión del desarrollo desde la endogeneidad, como proceso que surge desde los territorios, pero que es estimulado, condicionado, regulado y protegido desde el Estado Nacional. Fórmula que pudiera ser aplicada en el ámbito de las relaciones internacionales, si el sistema de globalización existente no estuviera condicionado por el esquema neoliberal.

Sólo así podrá lograrse una tendencia a la nivelación del desarrollo de los sistemas territoriales y será posible una gestión desde la endogeneidad, que disminuya paulatinamente las condiciones de dependencia de los territorios periféricos, condicionada históricamente.

Se puede concluir que cuando existan procesos de gestión del desarrollo que no creen las condiciones para que todas estas fuentes se pongan de manifiesto, se estarán limitando las posibilidades de desarrollo del territorio y profundizando sus deformaciones estructurales. Vista esta problemática, se deben estudiar detenidamente los factores condicionantes de la endogeneidad.

\subsection{Los factores del desarrollo desde la endogeneidad}

Las teorías del desarrollo endógeno han venido tomando cuerpo a partir de la nueva concepción del espacio, como un entramado de agentes y elementos económicos, históricos, sociales, culturales, políticos e institucionales, que poseen modos de organización y de regulación específicos (Mella Márquez, 1999).

Es por ello que se considera como el primer factor del desarrollo desde la endogeneidad al elemento más dinámico de los que intervienen en el proceso, o sea, las personas que encarnan los diferentes actores del desarrollo. En ellas se materializa y toma cuerpo el sistema de relaciones socioeconómicas del territorio, y son las portadoras de toda la dinámica de generación de riquezas, conocimientos, tecnologías, iniciativas y soluciones para los problemas del desarrollo.

Por tanto, el primer objetivo de la gestión del desarrollo desde la endogeneidad ha de ser la creación de mecanismos para lograr la participación activa de todas las personas ${ }^{9}$ como actores del desarrollo. Sólo así comienzan a crearse condiciones para que

\footnotetext{
${ }^{9}$ Existen territorios que su principal limitante lo constituye el factor humano, en los cuales tendrían una relevancia considerable todos los mecanismos encaminados a favorecer la estabilidad en el crecimiento demográfico, las migraciones, la formación y capacitación profesional y una mayor inclusión social.
} 
el territorio deje de ser un simple soporte físico o espacial de la actividad vital de la sociedad, y se convierta en un elemento activo del desarrollo, elemento éste que constituye la base de la respuesta a la concepción del desarrollo como un proceso de transformación social, y de despliegue de todo el potencial humano.

Estos mecanismos pueden ser del tipo económico, de manera que cada cual perciba que recibe según los resultados que entrega a la sociedad. Es por ello que en la gestión del desarrollo desde la endogeneidad, se debe propiciar que cada cual reciba el efecto de los resultados de su trabajo a través de mecanismos de producción, distribución y redistribución, lo más coincidentes posibles tanto en tiempo como en espacio. En este sentido motivacional, tienen gran importancia también los mecanismos del tipo movilizativo o formativo, como pueden ser los procesos de capacitación, que permiten elevar la identidad territorial, la unidad nacional, la cultura general y del desarrollo y la cultura de gestión de los diferentes actores y la población. Para ello, resulta necesaria la existencia de sistemas de capacitación, superación y aprendizaje, así como sistemas de divulgación que contribuyan a crear esta cultura de desarrollo.

Otro factor que condiciona y produce efectos sinérgicos en los territorios lo constituye la estructuración y funcionamiento del sistema productivo territorial (Vázquez Barquero, 1999: 38), pero entendiéndose como tal, no solamente al conjunto de empresas locales, sino al entramado productivo y de servicios del territorio, que estructurado en un sistema reticular formará un sistema flexible que a través de la externalización, la subcontratación y el vínculo total de sus actividades productivas y de servicios, genere economías de escala y efectos de aglomeración (Bellandi, 1986) permitiendo que se produzcan procesos de crecimiento económico y generación de un efecto de difusión en todo el territorio.

Cuando este sistema se interconecta a través de interfaces con el sistema de innovación tecnológica, la difusión de la innovación y el conocimiento se enriquecerán las posibilidades de dicho sistema, con lo que se logrará que las estrategias interactivas de difusión tecnológica vayan ocupando un espacio más prominente ante las estrategias lineales que se venían aplicando (Vázquez Barquero, 2000).

Esto implica que el sistema de gestión deberá contribuir a la estructuración de redes de empresas, que propicien la circulación de bienes y servicios, de flujos financieros y de tecnología 
hacia el interior del territorio, alimentando así su potencialidad. La gestión del desarrollo desde la endogeneidad se logrará en la medida en que se propicien los vínculos entre las empresas del sistema productivo del territorio, ya sean locales o grandes empresas externas ubicadas en el territorio.

Para que estas sinergias se pongan de manifiesto, resulta preciso una convergencia entre las estrategias del territorio, de la red en su conjunto y de las empresas incluidas en ellas, lo que requiere del sistema gestor del desarrollo una capacidad de gestionar sistémicamente, para crear las relaciones que sean necesarias para dinamizar los flujos comerciales, financieros, de información y conocimientos y establecer los espacios de concertación y así lograr que todo el sistema se aprecie como una unidad que busque la elevación de la competitividad del territorio en su conjunto.

La teoría clásica del desarrollo endógeno concibe que estas redes se forman de manera espontánea como una respuesta productiva de los territorios ante los retos de la globalización (Vázquez Barquero, 2000). Y si bien existen evidencias empíricas de que en determinados casos esta respuesta productiva se encuentra en algunos territorios, y que sus potenciales de desarrollo e historia productiva lo propician, existen otros territorios en los cuales la situación es tal que, de manera independiente, no es posible crear dicha capacidad.

Resulta necesario, pues, que la generación de redes de empresas en el territorio sea gestionada y estimulada por políticas inductoras de cooperación y flexibilidad en la estructura del sistema empresarial, y de manera particular para el caso cubano, que los gobiernos locales tengan una capacidad de generación de estas redes y de su adecuada gestión a partir de políticas locales, compatibilizadas con el gobierno central.

Otro factor lo constituyen los flujos de innovación, en los cuales la sinergia se produce de manera similar a lo que ocurre en una tormenta de ideas, cuando un grupo se encuentra trabajando en la solución de un problema. En ese caso, una idea se fertiliza con otra, complementándose y creando una solución incremental compartida. Asimismo, la red de innovación incremental se fertiliza y complementa y va creando condiciones para elevar la competitividad de ese sistema productivo territorial. Como resulta lógico, éste constituye uno de los elementos productores de sinergias que requiere de mayores condiciones del potencial interno del territorio, o de impulsos exógenos, lo que significa que 
para territorios deprimidos de la periferia no será fácil crear estas redes de innovación, ni mucho menos convertirlas en entornos innovadores (Furio Blasco, 1996 y Maillat, 1995). Esta dependencia de impulsos externos requerirá de instrumentos organizativos y de protección, que impidan la generación de procesos de dependencia tecnológica.

Vale destacar que estas aglomeraciones (redes o entornos innovadores) requerirán una unidad de los actores, así como una confianza y cooperación que haga viables los flujos incrementales de innovación. Resulta importante destacar el extraordinario significado que tiene la asimilación de estrategias interactivas de innovación, para lo cual resulta indispensable la existencia de interfases en el territorio que las faciliten y sean portadoras de esta interacción entre el desarrollo científico y tecnológico y los sistemas productivos de los territorios.

Por otra parte, como plantea Carlos de Mattos:

...desde el punto de vista de los factores privilegiados por las nuevas teorías del crecimiento, los lugares que disponen de mejor dotación de capital físico, capital humano y conocimientos, serían los de mayor potencial endógeno y, por tanto allí, donde dadas las externalidades positivas de estos factores y su incidencia mancomunada condicionaría las posibilidades de acumulación y crecimiento de cada territorio (De Mattos, 1999).

Es por ello que las ciudades constituyen el espacio más atractivo para el establecimiento de los sistemas productivos, donde se nutre de los diferentes factores productivos, relacionándose con la comunidad e interactuando con ella. Cuando las acciones de gestión provocan una adecuada organización y estructuración de la comunidad y de los espacios urbanos donde radican -cualquiera que sea su nivel en la jerarquía en el sistema de ciudades- se logran efectos sinérgicos, tales como:

- Las economías de aglomeración se producen en la medida en que aumenta la jerarquía de la ciudad;

- La posibilidad de acceso directo a proveedores y clientes con reducidos costos de transporte y de transacción;

- La existencia y desarrollo de infraestructuras productivas y de servicios, que permiten el logro de externalidades en el sistema productivo del territorio y permiten diversificar el espacio urbano.

Otro elemento decisivo de la ciudad como factor del desarrollo endógeno radica en el aprovechamiento, explotación y enrique- 
cimiento del patrimonio cultural del territorio, de los cuales las ciudades constituyen un sitio ideal de localización, lo que permite además la manifestación de toda la riqueza cultural, espiritual y humana del hombre. Lo planteado no significa que los procesos endógenos sólo sean posibles en los espacios urbanos, sino que la ciudad es un factor condicionante de desarrollo endógeno en los territorios.

Todos los factores analizados anteriormente tendrán diferentes grados de madurez y de posibilidades de manifestación, en dependencia del potencial de desarrollo existente en los territorios. No puede concebirse que estos factores se manifiesten de igual forma en el Valle del Silicón en Estados Unidos o en el municipio de San Miguel del Guaporé, en el estado de Rondonia, centro de la Amazonia brasileña, o inclusive en el municipio de La Habana Vieja o el municipio de Najasa, en la provincia cubana de Camagüey.

La gestión del desarrollo desde la endogeneidad persigue hacer dinámicas las fuentes internas de sinergias de los territorios, que son capaces de generar crecimiento económico, elevación del bienestar material y espiritual y ofrecer posibilidades de progreso, en armonía con el entorno natural del territorio, y ha de ser gestionado a través de un sistema de políticas que induzcan la generación de sinergias portadoras de desarrollo endógeno.

Como se vio, todo este sistema de gestión dependía de la existencia de los vínculos necesarios entre todos estos procesos y todo ello dependerá de la diversidad, de la estructura y de las particularidades económicas y sociales del sistema institucional que condiciona el sistema socioeconómico vigente en la sociedad.

La envoltura institucional condiciona la forma en que el progreso y el bienestar se gestionan; distribuye y llega a los individuos de la sociedad a partir de los diferentes mecanismos y principios éticos en que se sustentan los distintos sistemas socioeconómicos. El sistema institucional debe tener una riqueza de mecanismos e interfaces, que garantice en el ámbito territorial la existencia de los vínculos necesarios para que se produzcan relaciones horizontales en el ámbito del territorio y difundan el desarrollo hacia su interior.

Uno de los aspectos más polémicos en lo referente a las instituciones lo constituye el papel que juega el Estado en la sociedad, y sobre todo la capacidad que éste tiene para dirigir la 
gestión del desarrollo. Por ello, la definición y establecimiento concreto del actor que va a ejecutar la gestión del desarrollo depende íntegramente de los juicios de valor con que se encierra el concepto de desarrollo endógeno.

Es necesario destacar que todas las teorías y los modelos de crecimiento y desarrollo endógeno que se han elaborado en los últimos años parten de la base de que el Estado y sus políticas tienen que operar sin introducir distorsiones en el libre juego de las fuerzas del mercado. Este planteamiento se realiza sobre la base de lograr que:

...la economía local se autorganice, de forma espontánea, guiada por la necesidad de las empresas de producir bienes y servicios para un mercado exigente en el que compiten con los productos de empresas de otras ciudades y regiones (Vázquez Barquero, 1999: 34).

No obstante, el desarrollo de estas teorías ha ido reconociendo un papel relativamente más importante al Estado en el logro de estos propósitos de endogeneidad. Carlos de Mattos (1999) presenta un análisis referencial de este criterio cuando resume:

"Así pues, distintos autores vinculados a esta corriente han esbozado una concepción general sobre el tipo de Estado que consideran compatibles con sus planteos", estableciendo que "la aparición de gobiernos que garanticen los derechos de propiedad físicos e intelectual, que regulen el sector financiero y exterior y eliminen las distorsiones, y que mantengan un marco legal garante del orden es deseable", siendo éste el contexto en el que se reconoce que el gobierno "...juega un papel importante en la determinación de la tasa de crecimiento a largo plazo" (Sala i Martín, 1994).

Este autor destaca criterios que justifican la intervención pública orientada a asegurar el mejor despliegue de las fuerzas del mercado: la gestión de las externalidades y la provisión de ciertos bienes públicos, con el propósito, en última instancia, de generar en cada lugar un ambiente económicamente atractivo para la inversión privada.

Lo anterior viene a reforzar la idea de que el Estado debe tener un papel de importancia creciente en cualquier circunstancia institucional. No obstante, no es objetivo del presente trabajo ofrecer soluciones universales a esta problemática, sino despejar en qué medida esta lógica de pensamiento es aplicable para Cuba.

El Estado y el gobierno cubanos han mantenido y mantienen la capacidad de liderar las transformaciones a que viene siendo sometido; esto constituye el elemento de mayor vitalidad, ca- 
pacidad y poder de convocatoria en el territorio para liderar los procesos de gestión del desarrollo, lo que además le permitirá mantener el control de las transformaciones de la economía y la sociedad.

Pero, como se desprende de lo ya dicho, la participación activa de los órganos locales de gobierno en la gestión del desarrollo no puede lograrse desde una posición totalmente centralizada, sino que requiere de procesos descentralizadores que ofrezcan la posibilidad de activar, desde la base, las fuerzas productoras de sinergia, combinado con la acción centralizada encargada de la regulación general de las proporciones sectoriales y territoriales de la economía nacional y de los mecanismos de inversión, distribución y redistribución que para ello sean necesarios.

Por tal motivo, todo este proceso debe ser aplicado sobre la base de funcionamiento de la planificación, como elemento encargado de establecer el vínculo entre los propósitos territoriales y sus capacidades, armonizándolas con el resto de la economía nacional.

\section{La gestión del desarrollo regional. Un nuevo enfoque para la economía cubana}

La gestión del desarrollo en Cuba fue una de las premisas que guió la acción del Estado a partir del triunfo de la Revolución, en consonancia con la estructura organizativa y de carácter unitario que siempre tuvo el Estado cubano. Así, los procesos de desarrollo fueron organizados y ejecutados con una perspectiva centralizada, en la búsqueda de un desarrollo integral que permitiera resolver los enormes problemas que caracterizaban la sociedad cubana hasta 1959. En la medida en que se fue profundizando en el proceso revolucionario y en la institucionalización del Estado socialista, los métodos se fueron refinando en correspondencia con la aplicación de sistemas centralizados de planificación.

La planificación de la economía nacional, en sus diferentes variantes y métodos de ejecución, permitió a partir de 1960 estructurar un sistema que sentó las bases para un desarrollo armónico y proporcional en la distribución de las riquezas para todo el país. Este proceso, marcado por un enfoque "del centro al territorio", aprovechó las ventajas de recursos naturales, humanos, de infraestructura y capacidades instaladas que brindaban los territorios a las inversiones para el desarrollo. Sin embargo, y 
a pesar de los buenos propósitos, la planificación territorial se enfocaba (y se enfoca) desde una perspectiva nacional, pero con un incipiente enfoque de planificación territorial desde abajo, manteniéndose las desigualdades territoriales.

De igual forma, en la primera etapa de la planificación y aun en las condiciones actuales es limitado el enfoque sistémico de un territorio y de la planificación, al prevalecer objetivos de ordenamiento territorial con predominio de la planificación sectorial y planes que sólo involucran a la subordinación local con un alto grado de centralización. Existe, además, una perspectiva limitada en cuanto a considerar al territorio como elemento activo del desarrollo, contenido de potencialidades y generador de sinergias. En los años iniciales de la planificación en Cuba se reconocen (Rodríguez, 2000: 5) insuficiencias tales como las siguientes: Las categorías financieras desempeñaban un papel pasivo, al igual que la medición de los criterios de eficiencia basados en los mecanismos monetario-mercantiles. La asignación y control de recursos en términos físicos reducía la posibilidad de la participación de los trabajadores en el proceso de planificación y control de la actividad económica, existiendo rigidez en la elaboración y ejecución de los planes.

Los problemas persistieron hasta los primeros años de la década de los 80, cuando comenzó el periodo de rectificación de errores y tendencias negativas, que entre otras medidas se encaminaba al perfeccionamiento de la planeación.

Este proceso fue interrumpido por el Periodo Especial ${ }^{10} \mathrm{y}$ por la incertidumbre que caracterizaba y caracteriza el nuevo entorno de la economía cubana: estos nuevos acontecimientos eliminaron las bases sobre las que se sustentaba el plan de la economía: suministros seguros; precios estabilizados; planes quinquenales con los países socialistas, que permitían un balance de recursos, distribuirlos y redistribuirlos y que tenían primacía ante las categorías monetario-financieras, pero generaban ineficiencia en el uso de los recursos; subsidios adaptados al aumento de los costos de producción de las empresas públicas; poco aprovechamiento de los avances tecnológicos, etcétera. Se interrumpió, además, la planeación territorial a escala central y en las provincias se mantuvo la atención a la subordinación local (León Bilbao, 2000, 12).

${ }^{10}$ Etapa que comenzó con el derrumbe del campo socialista y colocó a Cuba en una situación de crisis. 
A partir de 1989, esta situación provocó un efecto resultante de las siguientes situaciones contrapuestas: por una parte, la infraestructura existente permitió establecer una estrategia de resistencia, que ofreció posibilidades reales de enfrentar la crisis que se desarrolló a partir de ese momento y que tuvo su momento más crítico en el año 1993, pero, por otra parte, puso de manifiesto que no se habían desarrollado todas las capacidades internas que existían en el país, y que era necesario establecer los mecanismos que permitieran poner en marcha todas esas reservas.

A partir de este momento el país se enfrentó a una "estrategia de resistencia que diera coherencia lógica a las medidas adoptadas" (MEP, 2001) encaminada a frenar el descenso de la economía, reordenarla en las nuevas condiciones y adoptar un grupo de políticas económicas (entre 1992 y 1994 ), con lo que se dio lugar a un proceso de descentralización de decisiones.

En 1990 comenzó un nuevo periodo para la economía cubana, marcado por la aplicación de un grupo de importantes medidas, que han ejercido un impacto en la situación económica y social del país, encaminadas a transformar la estructura y el funcionamiento de la economía. Entre las transformaciones efectuadas y sus impactos más importantes sobre la economía y la sociedad cubanas, se destacan las siguientes:

- Se le ofreció un mayor impulso al turismo, permitiendo aprovechar su dinamismo, su capacidad de generar ingresos en divisas en un breve plazo y sus posibilidades de influir en la reanimación de otros sectores.

- Se crearon las Unidades Básicas de Producción Cooperativa (UBPC), con el propósito de incentivar la producción agropecuaria, a partir de una modificación de las condiciones de trabajo e ingreso de los obreros agrícolas, debido a una mayor personalización de los resultados del trabajo, buscando un vínculo más directo entre la producción y la distribución de los resultados.

- La ampliación del Trabajo por Cuenta Propia, con el objetivo de crear alternativas de empleo en las difíciles condiciones de contracción de la economía y para ofrecer cauce legal al trabajo de personas con posibilidades de incrementar la oferta de bienes y servicios necesarios para la población. 
- La aplicación de un conjunto de medidas para lograr el saneamiento financiero interno, a través de la descentralización en la ejecución del presupuesto; la elevación de los precios de los productos no esenciales, como la bebida y el tabaco; la eliminación del subsidio a las empresas no rentables y la introducción de un nuevo sistema tributario, anteriormente casi inexistente.

El impacto de estas transformaciones ha permitido a Cuba enfrentarse a las enormes presiones políticas que recibe desde el exterior y crear una capacidad de resistencia por sí sola. Como resultado de este proceso de transformaciones, la economía cubana ha sufrido cambios que por su naturaleza son estructurales, funcionales y organizativos, y llevan implícito un fuerte contenido descentralizador. Esto permite que puedan ser tomadas medidas organizativas encaminadas a lograr un acercamiento entre la obtención de la riqueza en los territorios y sus procesos de distribución y redistribución.

Los cambios estructurales e institucionales (aún no finalizados) hacen cada vez más complejo el sistema objeto de planificación, expresado en: la aparición de nuevos actores económicos (propiedad cooperativa, empresa mixta, sociedades mercantiles, trabajo privado no agrícola); adelgazamiento de la administración pública; circulación interna de un grupo de divisas extranjeras y la dualidad monetaria; reconstrucción del sector exportador; creación de nuevas entidades de servicios (bancos, agencias financieras) para el intercambio con los nuevos mercados; ensanchamiento del mercado interno; sustitución de métodos verticales de dirección administrativa por métodos de relaciones económicas horizontales entre actores (descentralización del sistema de gestión empresarial); aparición de mecanismos indirectos de regulación a través de la política fiscal, monetario-financiera y comercial; implantación de relaciones contractuales y de instrumentos de cobros y pagos en el sistema empresarial, entre otros.

Por otra parte, la economía cubana es una economía abierta al comercio exterior y no es posible ignorar las rupturas de las tendencias (una vez que de forma abrupta se perdieron las relaciones comerciales y financieras mantenidas durante décadas con los antiguos países socialistas) que se están produciendo a escala internacional, y que hacen el entorno más dinámico y con mayor grado de incertidumbre. 
Se introducen relaciones de mercado (aunque aún no es nítida la división del trabajo entre planificación y mercado), lo que necesariamente introduce la flexibilidad en la planeación y plantea de una nueva manera la relación plan-mercado en la economía cubana, cuestión que requiere de una solución teórica y práctica.

El país está obligado a saltar de un modo intensivo de crecimiento a otro dominado por los imperativos de la productividad, competitividad, especialización, tecnología y flexibilidad de adaptación al cambio (CEPAL, 2000), lo que requiere del uso de la planeación estratégica. ${ }^{11}$

Esto permitirá la aplicación y desarrollo de iniciativas locales, en la explotación al máximo de las ventajas comparativas de los territorios y que se logre la utilización de las economías externas que se generan en los sistemas productivos y en las ciudades; que se profundice en la aplicación del conocimiento y la tecnología en los procesos productivos, lo cual podrá influir en el logro de un crecimiento económico al crearse una mayor motivación y participación en todos los procesos.

Es por ello que en esta nueva etapa y en este nuevo escenario, lo territorial debe cobrar una nueva dimensión, no sólo porque aparecen nuevos objetivos intrarregionales desde el espacio subnacional, vinculados a los cambios producidos, sino porque desde éste se puede aminorar el impacto económico y social de las transformaciones antes mencionadas y, sobre todo, contribuir a promover el desarrollo del país y de los propios territorios, potenciando los factores endógenos presentes en los mismos, velando además por la integridad de estos procesos.

Este nuevo enfoque debe plantear la gestión del desarrollo como una combinación de la gestión desde abajo con la gestión desde arriba, en la que esta última juegue un papel de regulador del comportamiento global de la economía; de regulación de las corrientes exógenas de financiamiento que se asienten en el territorio, que propicie y estructure las condiciones para que las manifestaciones endógenas tengan lugar de la forma más plena posible, y para establecer los mecanismos de redistribución, que permitan reducir las disparidades del desarrollo hacia dentro de la economía cubana, influyendo, de forma sistemática, en el incremento de la atractividad y el aprovechamiento y reforzamiento del potencial de desarrollo de los territorios.

${ }^{11}$ Primero en el ámbito empresarial y posteriormente, de forma incipiente, en el ámbito territorial público. 


\section{Premisas para la gestión del desarrollo desde la endogeneidad en el caso de la economía cubana}

Para lograr la gestión desde los territorios, resulta necesario el cumplimiento de un conjunto de premisas que hagan posible poner en juego mecanismos que permitan la gestión del desarrollo desde la endogeneidad.

El carácter centralizado del sistema de dirección de la economía cubana ha generado una tendencia a la autarquía de los diferentes elementos del sistema, lo que provoca, en los territorios, una tendencia a la atrofia del sistema de relaciones interempresariales, que disminuye la eficiencia proveniente de la división social del trabajo y genera enclaves productivos y de servicios que limitan las posibilidades de reinversión directa de parte de la riqueza producida en el territorio, elemento que constituye una condición básica de la elevación de la capacidad de desarrollo endógeno de los mismos y de una elevación de su grado de participación de los distintos actores en su gestión.

Una de estas premisas es la participación más activa de los órganos locales de gobierno en la gestión del desarrollo, lo cual no puede lograrse desde una posición totalmente centralizada, sino que requiere dar continuidad a los procesos descentralizadores que ofrezcan la posibilidad de activar, desde la base, las fuerzas productoras de sinergias, en combinación con la acción centralizada, encargada de la regulación general de las proporciones sectoriales y territoriales de la economía nacional y de los mecanismos de inversión, distribución y redistribución necesarios.

El sistema de gestión que se diseñe para aplicar este enfoque a la economía cubana debe partir del análisis de un conjunto de particularidades de su sistema de dirección, de manera que la capacidad endógena de desarrollo de los territorios se manifieste a partir de las condiciones concretas del sistema institucional cubano. Primeramente, porque la dinámica de acumulación de la economía cubana mantiene la preeminencia de la propiedad estatal, lo que requiere formas de motivación y estimulación de la creatividad, ajenos a la dinámica ciega de las fuerzas del mercado, aunque sin negar la posibilidad de la existencia de relaciones de mercado. En segundo término, porque el sistema de dirección de la economía cubana se encuentra sometido a procesos de cambio con un fuerte contenido descentralizador, que combina diferentes formas de descentralización, incluida la territorial, las cuales 
deben alcanzar un nivel de profundidad tal que se logre un equilibrio entre la descentralización funcional del sistema empresarial -que supone la aplicación de los programas de Perfeccionamiento Empresarial ${ }^{12}$ y la territorial que se pueda manifestar a través de los gobiernos locales. Y tercero, porque el papel de las estructuras de gobierno en la gestión del proceso de cambio que en estos momentos se está acometiendo debe incluir la creación de los mecanismos necesarios que los capacite para realizar la gestión del desarrollo desde la endogeneidad, y además, para que se cree una cultura de gestión del desarrollo desde la base.

Para Cuba resulta necesario un esquema de gestión del desarrollo que sea una combinación entre las potencialidades territoriales, la competitividad regional, los mecanismos de regulación estatales y de redistribución de las riquezas (para mantener el propósito de avanzar en la reducción de las disparidades territoriales del desarrollo) y el logro de la inserción competitiva de los territorios en el proceso de globalización.

Por ello, una de las premisas está dada en la estructuración del sistema territorial, de manera que se fomenten las corrientes financieras desde los territorios, para lo cual, en las condiciones de Cuba, se requiere de procesos descentralizadores y de negociación con los niveles centrales, para que los gobiernos locales alcancen una capacidad de decisión suficiente sobre parte de la riqueza creada en los mismos, después de haber cumplido con los aportes a los niveles centrales.

Los mecanismos que hacen viable esta premisa propician la creación, por parte de los gobiernos locales, de una capacidad financiera local destinada a la ejecución de procesos inversionistas, asociados al fortalecimiento del sistema productivo del territorio, o la red de aseguramiento a servicios sociales en el mismo y sus localidades. Esto permitirá extraer el máximo a la dinámica de acumulación de un sistema económico como el cubano y contribuirá a la ejecución de la gestión del desarrollo desde la endogeneidad.

Estas interfases pueden ser, por ejemplo, empresas comercializadoras y de servicio que logren materializar los vínculos intersectoriales de diferentes entes del sistema empresarial del territorio. Un ejemplo de ello lo constituyen las empresas

\footnotetext{
12 Estrategia de cambio organizacional que se aplica al sistema empresarial cubano, con un fuerte contenido descentralizador, desde el punto de vista funcional, y que se aplica como una extensión de las experiencias obtenidas, en un proceso similar, por el sistema empresarial de las Fuerzas Armadas Revolucionarias.
} 
comercializadoras que puedan garantizar los vínculos entre el turismo y la agricultura en los territorios, y donde las relaciones de intercambio se produzcan tomando en consideración la situación de los mercados locales de productos agropecuarios y las exigencias de calidad y precios que plantea el turismo (Hernández, 1999). ${ }^{13}$

También pueden asumir diversas formas como Patronatos (como algunas de las funciones que realizan hoy las Oficinas de los Historiadores en varias provincias del país y que constituyen una corroboración palpable de los planteamientos centrales de este trabajo). El funcionamiento y los resultados de estas oficinas constituyen un ejemplo fehaciente de las tesis fundamentales de este trabajo, al validar la posibilidad de que en un sistema institucional como el de Cuba se pueden crear procesos de desarrollo desde la endogeneidad. ${ }^{14}$

Otra modalidad son las agencias de desarrollo (como algunas de las funciones que realiza el Ministerio de la Inversión Extranjera y la Colaboración), que pueden constituir un ejemplo de las vías que tendrá el gobierno central de regular las relaciones del territorio con el sector externo. Otras podrían estar alimentando el presupuesto a partir de las Oficinas de Administración Tributaria y las Direcciones de Finanzas de los Territorios y los distintos mecanismos de financiación del desarrollo que puedan provenir de los programas sectoriales de la economía nacional, en dependencia del objeto de desarrollo que se quiera estimular y las características del potencial de desarrollo del territorio.

Todas estas entidades, bajo la dirección de los órganos locales de gobierno, constituyen al mismo tiempo un mecanismo apropiado para que el gobierno local estructure y apruebe un proceso inversionista, orientado desde la endogeneidad, encaminado al logro de la gestión del desarrollo. Resulta muy importante destacar la necesidad de gestionar el vínculo de estas entidades

\footnotetext{
${ }^{13}$ Este trabajo propone la creación de una empresa comercializadora de productos agrícolas, que sirva de interfase entre las Cooperativas de Producción Agropecuarias y las empresas turísticas del territorio, así como para hacer viable los procesos inversionistas que deban elevar la competitividad de la producción agropecuaria.

${ }^{14}$ Los autores realizaron un proceso de investigación en las Oficinas de los Historiadores de la Ciudad de la Habana (OHCH) y la de Camagüey (OHCC), las que con diferentes grados de desarrollo y complejidad, dadas la madurez de los proyectos respectivos y el potencial de desarrollo de partida, ofrecen un panorama alentador en cuanto al desarrollo de formas de captación y uso financiero generado en el territorio; al desarrollo de las redes empresariales a ellas asociadas; sus vínculos con los gobiernos de los municipios donde se desarrollan y el impacto social que van teniendo al convertirse en poleas de transmisión entre el desarrollo de su red empresarial y el desarrollo social de la comunidad de su área de demarcación.
} 
y el sistema empresarial con el desarrollo social del territorio y su comunidad, ya que éste ha de ser uno de los factores más importantes de estímulo a la participación.

Es necesario que los procesos de distribución y redistribución se acerquen lo más posible en tiempo y espacio a los productores directos de la riqueza material, de manera que se perciba, con la mayor nitidez posible, que cada cual aporta según su capacidad y recibe según su trabajo (Marx, 1878).

Por otra parte, para que exista un verdadero desarrollo desde la endogeneidad, es necesario prestar atención a lo que pudiéramos llamar los factores intangibles del desarrollo y establecer un sistema de información acerca del entorno en todas sus dimensiones.

Se hace necesario combinar la asimilación y difusión de la tecnología proveniente desde fuera del territorio y que pueda tener una carácter radical, con el nuevo paradigma tecnológico (innovación incremental), que significa la difusión y aplicación de pequeñas, pero constantes innovaciones y racionalizaciones que surgen de los esfuerzos e iniciativas de los actores locales, que al constituir soluciones propias, se convierten en ventajas competitivas de los territorios. El proceso de innovación tecnológica desde la endogeneidad se imbrica necesariamente con la utilización de los mecanismos ya existentes (Forum de Ciencia y Técnica) y la creación de otros nuevos, que permitan potenciar las reservas tecnológicas y productivas territoriales. Tal es el caso de la formación de oficinas de gestión de información y conocimientos, que integradas directamente a empresas o redes de empresas del sistema empresarial puedan ofrecer un nivel de información adecuado para la gestión de proyectos y financiamientos que redunden en la elevación de la competitividad del sistema empresarial y del territorio o localidad en su conjunto.

Un ejemplo de lo anterior es el papel que las universidades pueden jugar como interfases entre el desarrollo científico-técnico y tecnológico y los sistemas productivos de los territorios, y como dinamizadoras de estrategias interactivas del desarrollo científico, técnico y tecnológico llevado a la práctica.

Se requiere, además, dar continuidad al sistema de capacitación para los líderes de las estructuras locales y del sistema empresarial, que debe ser complementada con la creación de una cultura de gestión del desarrollo en la comunidad regional, con un reforzamiento del sentido de pertenencia regional y una imagen regional auténtica, para lo cual cada ciudad y cada localidad 
debe concebir programas de imagen que eleven el sentido de pertenencia con el territorio y propicien la participación activa de las ciudades y de su comunidad en la transformación del territorio.

Un sistema de dirección que pretenda mantener y desarrollar las conquistas que en el terreno social ha alcanzado, así como proyectar niveles superiores de eficiencia, debe lograr que la planificación continúe siendo la esencia del sistema de relaciones de producción y su sistema institucional, pero sobre la base de un mecanismo flexible que armonice dos procesos: por una parte, que le proporcione a los territorios un papel activo en la definición de los objetivos estratégicos, y por otra, esta planificación se debe realizar sobre la base de una integración de estas metas territoriales, con las que emanan de las metas sectoriales y nacionales, previstas y proyectadas desde el centro.

En lo relacionado con la problemática del equilibrio sectorial-territorial, es necesario que se establezcan los mecanismos que hagan posible que el desarrollo sectorial que emana del nivel central, a la vez que se una a las fuentes exógenas del desarrollo, no sea generador de enclaves productivos de bienes y servicios, sino que estas grandes empresas y sus estrategias sectoriales se integren armónicamente al sistema productivo territorial. Este aspecto requiere de una extrema atención por parte de los gobiernos locales, para que establezcan, definan y propongan cambios que permitan la creación de diferentes interfases, como las ya descritas, y que garanticen la potenciación de los vínculos horizontales en el territorios, en lo cual el sistema de planificación debe jugar un papel primordial.

La problemática medioambiental y de la protección de los recursos naturales y patrimoniales constituyen elementos que han sido atendidos exhaustivamente desde el centro y desde el territorio; no obstante, la acción local debe continuar el establecimiento de vías que profundicen la incorporación de esos recursos al desarrollo, y articulando la estrategia ambiental con el resto de los planes del territorio

Todos los procesos descentralizadores requieren de un riguroso equilibrio y control financiero (Fernández, 1999), ${ }^{15}$ partiendo de que los presupuestos locales adquirirán una mayor complejidad, al poseer fuentes de autogestión financiera, que

${ }^{15}$ Este trabajo propone métodos de análisis financieros con un enfoque microeconómico, que hacen posible la realización de análisis financieros con criterios de eficiencia y eficacia. 
requerirán de un riguroso control presupuestario y de planificación, de manera que exista equilibrio entre los objetivos propuestos y las posibilidades financieras del territorio.

Esta última premisa se logra, básicamente, a partir de la aplicación de una planificación estratégica territorial que integre en objetivos estratégicos territoriales todas estas dimensiones y garantice los equilibrios requeridos.

La experiencia de seis años en la implantación de la planificación estratégica en la provincia de Camagüey pone de manifiesto que resulta imprescindible armonizar y coordinar la planificación desde abajo, desde el punto de vista del ordenamiento físico-territorial (planes de ordenamiento territorial), desde el punto de vista técnico-económico (plan de la economía) y desde el punto de vista financiero (presupuesto), elaborados en un sistema de objetivos territoriales, con una visión al mayor horizonte de planificación posible, como plan estratégico territorial integrado.

Esta metodología de planificación sobre una base prospectiva ofrece el marco y los mecanismos apropiados para lograr el nivel de flexibilidad necesario, que permita el balance adecuado entre los diferentes mecanismos que utilice el sistema de relaciones económico-organizativas de la sociedad, y canalizar la participación de las estructuras de base en la gestión del desarrollo regional.

Como se puede apreciar, sobre la base del cumplimiento de las anteriores premisas deben ser diseñados los mecanismos que propicien la manifestación de este sistema de gestión desde la endogeneidad. Este proceso, liderado por las estructuras locales del gobierno, constituye una necesidad objetiva actual, para imprimir una nueva fuerza al proceso de cambio iniciado en 1986 en la economía cubana. Para ello, resulta necesaria la creación de mecanismos capaces de poner de manifiesto esta capacidad endógena de gestionar el desarrollo.

\section{Bibliografía}

Bellandi, M. (1986): "El distrito industrial en Alfred Marshall”, en Estudios Territoriales, núm. 20. La versión italiana de este trabajo fue publicada en La Industria, núm. 3, 1982.

Castro Ruz, F. (1971), La historia me absolverá, Editora Política, La Habana. 
Centro de Investigaciones de la Economía Mundial (CIEM) (1996), Informe de los resultados de la investigación sobre el Desarrollo Humano en Cuba, La Habana.

CEPAL (2000), La economía cubana, reformas estructurales y desempeño en los 90, Fondo de Cultura Económica, México.

De Mattos, Carlos (1999), Nuevas Teorías del crecimiento económico. Lectura desde la perspectiva de los territorios de la periferia, mimeo, Instituto de Estudios Urbanos, Pontificia Universidad Católica de Chile, Santiago de Chile.

Furio Blasco, E. (1996), Desarrollo territorial y procesos de innovación: Los milieux innovateurs, en Ciudad y Territorio, Estudios Territoriales, vol. XXVIII, núm. 110, pp. 639-649.

Fernández Andrés, Ana (1999), Enfoque analitico gubernamental para medir eficiencia y eficacia de empresas públicas y unidades presupuestadas, tesis de doctorado defendida en 1999. Publicación interna de la Universidad de Camagüey, Camagüey.

Hernández Companioni, José Carlos (1999), La relación intersectorial turismo-sector agropecuario en el contexto del desarrollo regional. El caso de la Provincia Ciego de Ávila, tesis de doctorado defendida en 1999. Publicación interna de la Universidad de Camagüey, Camagüey.

León Bilbao, Idalberto (2000), "El perfeccionamiento de la planificación y el papel del Estado en la Economía. Situación actual y perspectivas" en Seminario Nacional 40, Aniversario de la Planificación en Cuba, Ministerio de Economía y Planificación (MEP), La Habana.

Maillat, D. (1995), "Desarrollo Territorial, milieu y política regional” en A. Vázquez Barquero y G. Garofoli (eds.), Desarrollo Económico Local en Europa, Colegio de Economistas, Madrid.

Marx, Carlos (1878), Critica al Programa del Partido Social Demócrata Alema (Crítica al Programa de Gotha), Obras escogidas de Marx y Engels en tres tomos, Editora Revolucionaria, La Habana, 1970. 
Mella Márquez, J. M. (1999), "Evolución doctrinal de la ciencia regional: una síntesis”, en Economía y Política Regional en España ante la Europa del siglo XXI, Madrid.

Mep (Ministerio de Economía y Planificación) (2001), “Selección de materiales sobre teoría económica", VI Reunión del MEP, Centro de Estudios de Economía y Planificación, La Habana.

North, Douglass C. (1998), Institutions, institutional change and economic performance, Cambridge University Press.

PNUD (1990), Informe sobre el desarrollo humano, Tercer Mundo Editores, Bogotá.

Rodríguez, C. R (1983), Letra con filo, Editorial Ciencias Sociales, t. 2, La Habana.

Rodríguez, José Luis (2000), "Entrevista 40 años de planificación en Cuba", en El Economista, núm. 10, enero-febrero, La Habana, p. 5.

Sala i Martín, X. (1994), Apuntes de crecimiento económico, Antoni Bosch Editor, Barcelona.

Saxenian, A. (1994): Regional advance. Culture and competition in Silicon Valley and Route 128, Harvard University Press, Cambridge, Massachusetts.

Todaro, Michael (1984), El desarrollo económico del tercer mundo, t. 1, New York, pp. 35-44.

Vázquez Barquero, A. (1997): “Gran empresa y desarrollo endógeno. La convergencia estratégica de las empresas y territorios ante el desafío de la competencia”, en Revista EURE, vol. XXIII, núm. 70, Santiago de Chile, pp. 5-18.

Vázquez Barquero, A. (1999), Desarrollo, redes e innovación. Lecciones sobre el desarrollo endógeno, editorial Pirámide, Madrid. 
Vázquez Barquero, A. (2000), Desarrollo endógeno y globalización, en Revista EURE, vol. xxvi, núm. 79, Santiago de Chile, pp. 45-65.

Enviado: 7 de marzo de 2002 Aceptado: 13 de mayo de 2002 